\title{
JURISPRUDENCIA CONSTITUCIONAL EN MATERIA DE PROTECCIÓN DEL MEDIO AMBIENTE
}

(PRIMER SEMESTRE 2020)

\author{
CLARA ESTEVE JORDÀ \\ Investigadora Predoctoral Contratada Martí i Franquès \\ Universitat Rovira i Virgili
}


Sumario: 1. Nulidad de los preceptos legales que permiten la transformación urbanística de suelos integrados en la red natura 2000. 2. Competencias en materia de medio ambiente delimitadas por la legislación básica. Juicios de contraste. 3. Competencias sobre protección de animales. Falta de legimitación activa.

\section{NULIDAD DE LOS PRECEPTOS LEGALES QUE PERMITEN LA} TRANSFORMACIÓN URBANÍSTICA DE SUELOS INTEGRADOS EN LA RED NATURA 2000

En los últimos meses, el Tribunal Constitucional ha tenido que resolver hasta tres veces acerca de los preceptos legales que permiten la transformación urbanística de suelos integrados en la Red Natura 2000. En particular, sobre los terrenos de la comunidad autonómica de Extremadura. El territorio rural extremeño es extenso y encierra una gran diversidad paisajística, socioeconómica y cultural, con una escasa y dispersa población. De hecho, tal es su importancia en este sentido, que un treinta por cien de su superficie está bajo protección medioambiental. Así las cosas, la cuestión principal dirimida en este hilo de sentencias era si los suelos extremeños incluidos en la Red Ecológica Natura 2000 podían ser susceptibles de transformación urbanística o no, de acuerdo con la legislación autonómica.

Las SSTC 152/2019, de 25 de noviembre y 20/2020, de 10 de febrero provienen de una primera sentencia, la STC 134/2019, de 13 de noviembre, que fue la que determinó la inconstitucionalidad y nulidad del precepto autonómico regulador de la posibilidad de transformación urbanística de suelos protegidos. En ambas sentencias, tanto la fiscal general del Estado como la asociación CODAEcologistas en Acción reclamaban que se declarara extinguida la cuestión de inconstitucionalidad, por pérdida sobrevenida de objeto. Por contra, las representaciones de la Junta y de la Asamblea de Extremadura solicitaban la desestimación de la cuestión. Lo cierto es que sí existía coincidencia objetiva y argumental con la cuestión ya resuelta por la mencionada STC 134/2019, tanto por lo que se debatía en los procesos subyacentes como por los preceptos cuestionados. Por ello, el TC determina en ambas ocasiones que, en efecto, el precepto planteado había sido ya declarado inconstitucional y nulo por la STC 134/2019, de 13 de noviembre. Confirmando así que la expulsión del 
ordenamiento jurídico de un precepto determina la extinción de la cuestión, al haber quedado resuelta la duda planteada.

Analicemos, pues, la primera sentencia, que resulta determinante y concluyente para las dos posteriores. En la STC 134/2019, el Tribunal Constitucional resuelve la cuestión de inconstitucionalidad, por un lado, del art. 11.3.1.b), párrafo segundo, de la Ley 15/2001, de 14 de diciembre, de suelo y ordenación territorial de Extremadura, en la redacción dada por la Ley 9/2011, de 29 de marzo y, por otro lado, de la disposición adicional única de esta segunda ley.1

Concretamente, el primer precepto impugnado incluía, dentro de los terrenos que debían adscribirse al tipo de suelo no urbanizable, aquellos en los que concurrían valores "de carácter ambiental, natural y paisajístico". Además, añadía que la inclusión de un terreno en la red ecológica Natura 2000 no determinaba, por sí sola, su clasificación como no urbanizable. De modo que esto permitía la transformación urbanística de los terrenos en los términos que facultara la evaluación ambiental. Por su parte, la disposición adicional única de la Ley 9/2011, de 29 de marzo, establecía un procedimiento de homologación para la regularización de instrumentos de ordenación urbanística y territorial vigentes a la entrada en vigor de dicha ley - como se da en el supuesto de hecho, ante un proyecto de interés regional (PIR): una urbanización de lujo en una isla de 135 hectáreas en el embalse de Valdecañas (Cáceres) - a fin de declararlos conformes con esta modificación de la ley del suelo extremeña.

En la STC 134/2019, la cuestión queda parcialmente estimada, declarando el TC la inconstitucionalidad y nulidad del párrafo segundo del artículo 11.3.1.b), al resultar contrario a la normativa básica estatal, en su doble dimensión formal y material. Por un lado, se declara la vulneración de la Ley de sueloz y de la Ley de patrimonio natural y de biodiversidad.3 Por otro lado, también se declara inconstitucional conforme al derecho de la Unión Europea, que delimita la red

\footnotetext{
1 Ambas leyes quedaron derogadas por la posterior Ley 11/2018, de 21 de diciembre, de ordenación territorial y urbanística sostenible de Extremadura.

2 Los arts. 12.2 y 13.4 del entonces Real Decreto Legislativo 2/2008, de 20 de junio, que se mantienen sin cambios sustanciales en los arts. 13 y 21 del actual Texto refundido de la Ley de Suelo y Rehabilitación Urbana (Real Decreto Legislativo 7/2015, de 30 de octubre (TRLS).

${ }_{3}$ En concreto, el art. 46 de la Ley 42/2007, de 13 de diciembre, del patrimonio natural y de la biodiversidad
} 
Natura 2000,4 y a la infracción de los arts. 149.1.1 y 23 CE. La normativa estatal somete cualquier alteración de la delimitación del estado natural de los terrenos a la preservación de los valores ambientales, cosa que se estima incompatible con su transformación urbanística. Así, esta delimitación solo puede alterarse cuando lo justifiquen los cambios provocados por su evolución natural, científicamente demostrada, y a través del procedimiento establecido, que incluye un trámite de información pública y la aceptación de la propuesta de descatalogación por la Comisión Europea.

Por el contrario, se falla que la disposición adicional única de la Ley 9/2011 no es inconstitucional, pues no vulnera los arts. $117 \mathrm{ni} 118 \mathrm{CE}$. El TSJ de Extremadura alegaba que no se trataba de una disposición general, sino de una ley singular prevista para un caso específico, con la intención de eludir la ejecución de las sentencias recaídas en el proceso. Sin embargo, el TC lo rechaza, con el argumento de que "no se trata de una norma dictada en atención a un supuesto de hecho concreto y singular que agote su contenido y eficacia con él." Así pues, tampoco se vulnera el derecho a la tutela judicial efectiva del art. 24.1 CE, al no preverse una legalización automática, que impida el control por la jurisdicción contencioso-administrativa.

De este modo, tras el análisis del supuesto concreto, la conclusión que alcanza el Tribunal Constitucional parece ser favorable a una alta protección de los terrenos con valores ambientales recogidos en la Red Natura 2000, supeditando la utilización de dichos terrenos a la preservación de dichos valores, en beneficio del medio ambiente. Exige que el estado ambiental de dichos terrenos no transformados urbanísticamente, por lo menos, se paralice, lo que requiere que el planificador urbanístico les otorgue de forma ineludible la clasificación como suelo no urbanizable. Confirmándose pues, la prevalencia de la protección ambiental sobre el desarrollo urbanístico.

4 La Red Natura 2000 queda desarrollada en la Directiva 92/43/CEE del Consejo, de 21 de mayo de 1992, relativa a la conservación de los hábitats naturales y de la fauna y flora silvestres (Directiva hábitats). El segundo instrumento fundamental de la Red Natura 2000 es la Directiva 79/409/CEE del Consejo, de 2 de abril de 1979, relativa a la conservación de las aves silvestres, hoy sustituida por la Directiva 2009/147/CE del Parlamento Europeo y del Consejo, de 30 de noviembre de 2009 (Directiva aves). 
Sin embargo, cabe inferir que el voto particular que formula el magistrado don Ricardo Enríquez Sancho, nos vuelve a recordar ese dato revelador de la actualidad territorial extremeña. El magistrado alega que no se ha tenido en cuenta que la norma declarada inconstitucional afectará a todos los terrenos que en Extremadura estén integrados en la Red Natura 2000, que incluyen casi la tercera parte de todo el territorio de esa comunidad, impidiéndoles cualquier desarrollo urbanístico. Y en concreto, nos recuerda que el art. 46.5 de la Ley 42/2007 permite la ejecución de planes, programas o proyectos por razones imperiosas de primer orden, incluidas razones de índole social o económica. Para Enríquez, pues, la consecuencia tendría que haber sido la desestimación de la cuestión de inconstitucionalidad planteada, rechazando que la inclusión de un terreno en la Red Natura 2000 impida cualquier transformación urbanística.

A raíz de la STC 134/2019, de 13 de noviembre, el desarrollo urbanístico de los municipios extremeños cuyo territorio se encuentra incluido en la Red Natura 2000 queda en entredicho. Lo cierto es que, tal y como aducía la Sección Primera de la Sala de lo Contencioso-Administrativo del Tribunal Superior de Justicia de Extremadura, en su cuestión de inconstitucionalidad, un tercio del territorio extremeño está integrado en la red ecológica en cuestión, lo que dificulta el desarrollo urbanístico de sesenta y dos municipios. Hará falta ver si este fallo del Tribunal Constitucional resulta particularmente relevante para la resolución de eventuales problemas de desarrollo urbanístico en dicha región autonómica, o incluso para futuras cuestiones de transformación urbanística en otras comunidades autónomas.

\section{COMPETENCIAS EN MATERIA DE MEDIO AMBIENTE DELIMITADAS POR LA LEGISLACIÓN BÁSICA. JUICIOS DE CONTRASTE.}

En octubre de 2018, la Asamblea Regional de Murcia aprobó la Ley 10/2018, de 9 de noviembre, de aceleración de la transformación del modelo económico regional para la generación de empleo estable de calidad. La ley pretendía dar un impulso al modelo económico regional, mediante la homogeneización de los desfases legislativos naturales del paso del tiempo y "la adaptación de las necesidades de un tejido empresarial moderno y competitivo, abarcando todos los sectores productivos sobre los que se fundamenta el futuro desarrollo 
económico y social de la Región de Murcia”.5 Dicha ley, pues, de contenido heterogéneo, modificó hasta dieciséis textos legislativos regionales, entre otros, las Leyes de turismo,6 de ordenación territorial y urbanística7, y de protección ambiental integrada.8 La ley 10/2018 incluía, entre otras, medidas sobre sostenibilidad energética, intervención urbanística y medioambiental, con la finalidad de facilitar la actividad económica.

A raíz de esta aprobación, en marzo de 2019 más de cincuenta diputados y diputadas del grupo parlamentario confederal Unidos Podemos-En Comú Podem-En Marea, interpusieron un recurso de inconstitucionalidad contra la ley murciana. El recurso se tramitó, entre otras cuestiones, por razones de competencias de protección ambiental.9 En concreto, en la sentencia que deriva de este proceso, STC 161/2019, de 12 de diciembre de 2019, se enjuicia la constitucionalidad de los preceptos sobre mantenimiento y conservación de las obras de urbanización, incentivos de edificabilidad para la renovación hotelera, suelos protegidos, usos del suelo y protección ambiental. Varios son los preceptos impugnados que para esta crónica nos interesan, en materia de derecho ambiental.

En primer lugar, al art. 7.4 de la Ley 10/2018, que lleva por rúbrica "Incentivo de edificabilidad para la renovación hotelera" se le recriminaba que, al no establecer excepción alguna, el silencio de la norma podía dar paso a un incremento de edificaciones situadas en la zona de servidumbre de protección regulada en la Ley de Costas, lo que resultaría contrarío a la regulación estatal y a la doctrina constitucional en la materia. En relación con este precepto, el juez de la constitucionalidad considera que el art. 7.4 de la ley murciana no incurre en

\footnotetext{
5 Preámbulo de la Ley de la Asamblea Regional de Murcia 10/2018, de 9 de noviembre, de aceleración de la transformación del modelo económico regional para la generación de empleo estable de calidad.

6 Ley 12/2013, de 20 de diciembre, de turismo de la Región de Murcia

7 Ley 13/2015, de 30 de marzo, de ordenación territorial y urbanística de la Región de Murcia.

8 Ley 4/2009, de 14 de mayo, de protección ambiental integrada de la Región de Murcia.

9 El recurso no se impugnó exclusivamente por razones ambientales, sino alegando en primera instancia la vulneración de los principios de seguridad jurídica y autonomía local, recogidos en el art. 9 CE. El recurso alegaba que la ley 10/2018 adolece de falta de claridad, al ser un texto confuso, de difícil comprensión tanto para la ciudadanía como para las administraciones de deben aplicarla. Sin embargo, la contravención del principio de seguridad jurídica contenido en el artículo 9 CE fue desestimada.
} 
vulneración constitucional, siempre y cuando se interprete que el silencio de la norma impugnada no excluye los mecanismos estatales para compatibilizar el ejercicio de las competencias autonómicas con las medidas de protección del dominio público marítimo-terrestre. $Y$ añade que nada impide que resulte de aplicación la Ley de Costas estatal para el informe preceptivo y vinculante que debe emitir la administración estatal, en la elaboración de los planes especiales o estudios de detalle que pide la ley murciana. Así pues, con esta interpretación se evita la colisión de competencias estatales y autonómicas, si ambas actúan simultáneamente sobre la zona de la servidumbre de protección, "preservando así las prohibiciones que, por razones medioambientales ha establecido el Estado."

En segundo lugar, otro precepto impugnado era el apartado primero del art. 22 de la Ley 10/2018, al modificar la ley 13/2015, de 30 de marzo, de ordenación territorial y urbanística de la Región de Murcia y, supuestamente, violar la autonomía local constitucionalmente garantizada en los arts. 137 y $140 \mathrm{CE}$. Se le acusaba de posibilitar que los planes de ordenación territorial regularan los usos, obras e instalaciones de carácter provisional, y limitar así la competencia urbanística municipal. Sobre esta cuestión el Tribunal Constitucional desestima la impugnación, declarando que no se vulnera la autonomía municipal, porque las decisiones sobre los usos, obras e instalaciones de carácter provisional quedan para la regulación urbanística autonómica, advirtiendo que

A la comunidad autónoma corresponde diseñar el sistema jurídico de ordenación del territorio y utilización del suelo, el cual enmarca el ámbito en el que habrá de moverse el planificador local, lo que no representa vaciamiento alguno de las competencias urbanísticas locales sino prevalencia del interés supramunicipal que expresan los planes a los que se ordena jerárquicamente la planificación municipal. En ejercicio de dicha competencia, la previsión impugnada se sitúa, atendiendo a su contenido y ubicación sistemática, en el plano normativo, en concreto, el relativo al establecimiento de determinaciones básicas de carácter territorial vinculantes para ese mismo planificador urbanístico municipal. Tampoco obvia la actividad municipal sobre la asignación de dichos usos, con lo que no se merman las potestades de intervención y de vigilancia y control municipal. Finalmente, ha de advertirse que existe intervención municipal en 
la elaboración de estos instrumentos de ordenación, puesto que el art. 70.2 de la Ley 13/2015 prevé expresamente la audiencia a los ayuntamientos afectados por el plan [FJ 6].

Sobre el mismo art. 22, en el recurso de inconstitucionalidad se consideraba que su apartado noveno interfería en la normativa básica en materia de evaluación de impacto ambiental, pues privar a las normas transitorias de la consideración de instrumentos de planeamiento tiene incidencia en la normativa estatal medioambiental, en concreto, en los arts. 5, 6 y 8 de la Ley 21/2013, de 9 de diciembre, de evaluación ambiental. Sobre esta prescripción, el TC falla que, en efecto, deben declararse inconstitucionales y nulos ciertos incisos, al incurrir en contradicción con la normativa básica y ser contrarios al orden constitucional de distribución de competencias sobre medio ambiente. Por lo tanto, debemos interpretar a sensu contrario que el régimen jurídico de suspensión de la vigencia de los instrumentos de planeamiento urbanístico sí tiene la consideración de instrumento de planeamiento a efectos ambientales, aún no modificándose la clasificación prevista en el planeamiento. La legislación básica parte de que corresponde al órgano ambiental valorar en cada caso concreto los efectos significativos sobre el medio ambiente, a través de la evaluación ambiental estratégica simplificada. Y queda zanjada de esta forma la controversia competencial en materia de medio ambiente. Ello nos permite concluir que el TC, proteccionista y receloso a cualquier ampliación de regulación que interfiera sobre el ámbito medioambiental, aboga por la forma preventiva, y prohíbe que las normas autonómicas excluyan de evaluación ambiental determinadas categorías de planes y sus modificaciones que sí están sometidas a la misma de conformidad con la legislación básica. Y es que la exclusión de evaluación ambiental por parte de normas autonómicas sobre determinadas categorías de planes y sus modificaciones, supondría la reducción de los niveles mínimos de protección que sí establece la legislación básica y, por tanto, la vulneración de la competencia estatal en materia de medio ambiente.

En la misma línea, el art. 23.4 de la Ley 10/2018 también es acusado de rebajar el nivel de protección ambiental que otorga una norma básica dictada por el Estado en el ejercicio de sus competencias en materia de medio ambiente, contempladas en art.149.1.23 CE. Del mismo modo que en el precepto antes 
analizado, se plantea su inconstitucionalidad mediata o indirecta, pues se fija un criterio respecto a qué modificaciones no tienen carácter de sustanciales en las actividades potencialmente contaminadoras de la atmósfera, criterio que rebaja el nivel de protección ambiental fijado por el Estado en el ámbito de la autorización ambiental integrada. Sobre esta impugnación, el Tribunal Constitucional estima, mediante un juicio de contraste, que es posible salvar el precepto con una interpretación que resulte conforme al orden constitucional de distribución de competencias: debe entenderse que el art. 23.4 de la Ley 10/2018 no es aplicable a las actividades sometidas al régimen de autorización ambiental integrada, sino solamente a las autorizaciones a las que hace referencia la Ley de calidad del aire y protección de la atmósfera.10

La misma suerte corre la disposición adicional primera de la Ley murciana 10/2018. El TC interpreta que debe entenderse que la regularización de actividades no autorizadas, en el marco de la Ley 4/2009, de protección ambiental integrada, se refiere solamente a aquellas que no sean compatibles con la modificación del planeamiento exigida por Ley 12/2015, de 30 de marzo, de ordenación territorial y urbanística de la Región de Murcia, una vez que esa modificación haya sido aprobada inicialmente. Además, la Ley 10/2018 establece que ha de tratarse de actividades ajustadas a la legislación medioambiental y sectorial, a cuyo efecto habrán de recabarse la autorización ambiental autonómica y las demás sustantivas o sectoriales que sean exigibles, las cuales no quedan desplazadas ni excluidas por la autorización provisional de esta disposición adicional primera [FJ 8].

De este modo, y para concluir, en la sentencia 161/2019, de 12 de diciembre de 2019, se aprecia una vez más la tendencia proteccionista adoptada el guardián de la Constitución ante las competencias básicas de protección ambiental. En primer lugar, declarando la nulidad parcial de los preceptos legales autonómicos que introducen un incentivo de edificabilidad para la renovación hotelera y privan a las normas transitorias de la condición de instrumentos de planeamiento ambiental. Y en segundo lugar, permitiendo una interpretación conforme con la Constitución de los preceptos legales autonómicos que pueden incidir sobre la protección del dominio público marítimo-terrestre, afectan a las actividades 
potencialmente contaminadoras de la atmósfera y permiten la regularización de actividades carentes de licencia urbanística. Declarando, pues, la inconstitucionalidad solo en aquellos casos en que la contradicción es evidente, y salvando mediante juicios de contraste aquellos preceptos que por vía interpretativa pueden ser compatibles con la Constitución, cuando son efectivamente deducibles, de modo natural y no forzado [FJ 5.b].

\section{COMPETENCIAS SOBRE PROTECCIÓN DE ANIMALES. FALTA DE LEGIMITACIÓN ACTIVA}

La Sentencia 176/2019, de 18 de diciembre de 2019, debía resolver el recurso de inconstitucionalidad interpuesto por el Consejo de Gobierno de La Rioja en relación con diversos preceptos de la Ley del Parlamento de La Rioja 6/2019, de 26 de noviembre, de protección de los animales. En concreto, el promotor del recurso alegaba, en primer lugar, la vulneración de los arts. 8.1 .4 y 9.5 del Estatuto de Autonomía de la Rioja (EAR) y los arts. 149.1, 13 y 16 CE, al suprimir, la Ley riojana, los controles y garantías por razones de salubridad contenidos en la normativa básica estatal, la Ley 32/2007, de 7 de noviembre, para el cuidado de los animales en su explotación, transporte, experimentación y sacrificio, y el art. 20 de la Ley 8/2003, de 24 de abril, de sanidad animal. También se esgrimía que atentaba contra esta segunda ley la imposición de la esterilización forzosa de animales de compañía, pudiendo afectar a la propia evolución de las especies y causando pérdida de caudal genético importante, así como la señalización del traslado de animales de compañía vivos, contrariamente también a lo establecido en el Real Decreto 542/2016, de 25 de noviembre, sobre normas de sanidad y protección animal durante el transporte, dictado en ejecución de normativa europea.

Asimismo, la atribución a la consejería competente de la adopción de disposiciones para la adecuada gestión de las especies protegidas, entraba en colisión con lo dispuesto por la legislación básica estatal en materia de medio ambiente (Ley 42/2007, de 13 de diciembre, del patrimonio natural y de la biodiversidad, y Real Decreto 139/2011, de 4 de febrero, para el desarrollo del listado de especies silvestres en régimen de protección especial y del catálogo español de especies amenazadas), vulnerando así el art. 149.1.23 CE. También 
se alegaba que la imposición de autorización e inscripción registral y la llevanza de un registro oficial en los zoológicos era una reproducción de las normas estatales, que además delimitaba la eficacia de lo básico (STC 157/2004, de 23 de septiembre). Igualmente, la previsión de un régimen de infracciones y sanciones derivado de la contravención de las prohibiciones al maltrato de animales (art. 7), consideraba el Consejo de Gobierno riojano que era susceptible de tipificación de delito, vulnerando de esta forma la competencia exclusiva del Estado en materia de legislación penal, ex art. 149.1.6 CE. La enumeración de las infracciones graves y muy graves en la ley autonómica estaba deslegalizando la tipificación de conductas con cabida en delitos contra la fauna, toda vez que parecían convertirse en infracciones administrativas. Esto, pues, supuestamente provocaba inseguridad jurídica para el operador jurídico y para los administrados, al desconocer el alcance de su conducta. Con ello, alegaba el demandante, se vulneraban igualmente los principios de seguridad jurídica e interdicción de la arbitrariedad $y$ de legalidad penal (arts. 9.3 y $25.1 \mathrm{CE}$ ).

Sin embargo, todas estas cuestiones quedan sin resolver en esta sentencia, al declarar el Tribunal Constitucional la inadmisión del recurso de inconstitucionalidad, por falta de legitimación activa de su promotor, el Consejo de Gobierno de la Rioja. Su argumentación es que el art. 162.1.a) CE no puede interpretarse sino mediante "los márgenes que le permiten los términos literales del art. 32.2. LOTC" (STC 223/2006, de 6 de julio, FJ 2). La LOTC no atribuye legitimación para la impugnación de leyes propias a las mismas asambleas autonómicas que las aprobaron, y es esta la ley que especifica "la conexión entre los titulares de la acción de inconstitucionalidad y los posibles objetos de este, con lo cual el concepto de legitimación que el texto constitucional formula en términos muy amplios o genéricos adquiere su sentido técnico concreto" (STC 25/1981, de 14 de julio, FJ 2). Respecto de la legitimación activa, el TC reconoce la asimetría entre las cámaras de las Cortes Generales y las Asambleas Legislativas de las comunidades autónomas, dado que al segundo órgano, la Constitución no le atribuye legitimación a una determinada fracción del órgano parlamentario, sino al órgano en sí (art. 162.1.a) CE). Y es que, si no, estaríamos ante un conflicto entre órganos superiores de una comunidad autónoma. 
Conflicto interno que no corresponde resolver al Tribunal Constitucional. Esto advertido, el TC argumenta que la legitimación que pretende para sí el ejecutivo autonómico llevaría a romper el equilibrio o paridad institucional que debe existir entre uno y otro órgano superior de la comunidad autónoma para el ejercicio de la acción directa de inconstitucionalidad.

La cuestión de la legitimación activa de los gobiernos autonómicos para interponer recurso de inconstitucionalidad contra las leyes de sus propios parlamentos ha sido objeto de un amplio debate, tanto en la doctrina científica — como señala el magistrado Sr. Gonzáles-Trevijano: M.L. Balaguer Callejón, I. Torres Muro, R. Canosa Usera, M. Sánchez Morón y P. Pérez Tremps - como en resoluciones del propio Tribunal Constitucional. Ejemplo de esta discrepancia también lo demuestran los tres votos particulares que se emiten en la sentencia que analizamos, STC 176/2019. En el primer voto, el magistrado Juan Antonio Xiol Ríos se adhiere a los subsiguientes voto en su totalidad. El segundo voto particular es emitido por el magistrado Pedro José González-Trevijano Sánchez, al que se adhiere el magistrado Ricardo Enríquez Sancho. Finalmente, el tercer voto es emitido por la magistrada Maria Luisa Balaguer Callejón.

La opinión discrepante de estos magistrados merece ser comentada, pues la argumentación jurídica a la que se prestan no parece desacertada. Consideran los magistrados que debería haberse admitido la legitimación del Consejo de Gobierno de la Rioja, ya que la cláusula general de legitimación que contempla el art. 162.1.a) CE constituye una regla con una relación completa y cerrada de sujetos legitimados en todo caso. Dicho artículo recoge, de forma concisa, los sujetos legitimados para interponer el recurso de inconstitucionalidad: el presidente del Gobierno, el Defensor del Pueblo, cincuenta diputados, cincuenta Senadores y "los órganos colegiados ejecutivos de las Comunidades Autónomas y, en su caso, las Asambleas de las mismas". Esta enumeración taxativa no se remite a una posterior ley de desarrollo ni, por consiguiente, "habilitaría al legislador para introducir apreciaciones reductoras de la legitimación ad procesum" (Voto particular 2). Y para más inri, señalan los magistrados discrepantes, el subsiguiente art. 162.2 CE hace referencia específica a la facultad de la Ley Orgánica para determinar las personas y órganos legitimados "en los demás casos". Cosa que que permite deducir, a sensu contrario, que la 
Constitución estimaba suficientemente definidas las legitimaciones otorgadas a los sujetos previstos en el art. 162.1 a) sin necesidad de desarrollo posterior alguno. De este modo, se recrimina en los votos particulares que el art. 32.2 LOTC no tenía nada que matizarle al art. 162.1.a) respecto a la legitimación de los ejecutivos autonómicos para impugnar las normas emanadas de sus propias asambleas legislativas. Y se añade que la justicia será mejor cuanto más abierta y accesible esté a la ciudadanía y a los poderes públicos, colaborando con el control de constitucionalidad y los mecanismos de defensa de los derechos fundamentales.

Dicho todo esto, es una lástima, pues, que la constitucionalidad de la Ley del Parlamento de la Rioja, 6/2018, de 26 de noviembre, de protección de los animales, quede pendiente de resolución, desconociendo si invade competencias estatales o no, entre otras, en materia de medio ambiente y de protección y bienestar de los animales. Posiblemente habrá que esperar, en un futuro, a que otro promotor interponga un recurso de inconstitucionalidad análogo sobre la misma ley, que no podrá ser, en cualquier caso, el Consejo de Gobierno de la Rioja, mientras el TC no interprete la legitimación activa del art. 161.1.a) en otra dirección. 